Moving beyond domain-specific vs. domain-general options in cognitive neuroscience

\author{
Rie Asano ${ }^{\mathrm{a}, *}$, Cedric Boeckx ${ }^{\mathrm{b}, \mathrm{c}, \mathrm{d}}$, Koji Fujita ${ }^{\mathrm{e}}$
}

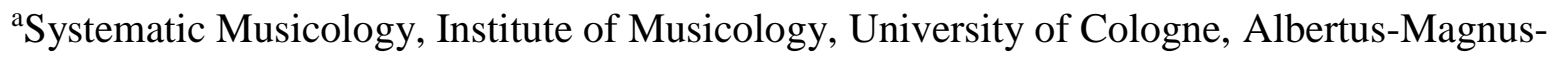
Platz, 50923 Cologne, Germany

${ }^{\mathrm{b}}$ Section of General Linguistics, University of Barcelona, Gran Via de les Corts Catalanes 585, 08007 Barcelona, Spain

${ }^{\text {c} U n i v e r s i t y ~ o f ~ B a r c e l o n a ~ I n s t i t u t e ~ f o r ~ C o m p l e x ~ S y s t e m s ~(U B I C S), ~ C a r r e r ~ d e ~ M a r t i ́ ~ F r a n q u e ́ s ~}$ 1, 08028 Barcelona, Spain

${ }^{\mathrm{d}}$ Catalan Institute for Advanced Studies and Research (ICREA), Passeig Lluís Companys 23, 08010 Barcelona, Spain

${ }^{\mathrm{e}}$ Graduate School of Human and Environmental Studies, Kyoto University, Yoshida, Sakyoku, Kyoto 606-8501, Japan

*Corresponding author: Rie Asano, Dr.

Department of Systematic Musicology, Institute of Musicology

University of Cologne

Albertus-Magnus-Platz

50923 Cologne

Germany

Phone: +49 (0)221 4705238

e-mail: rie.asano@uni-koeln.de 


\title{
Moving beyond domain-specific vs. domain-general options in cognitive neuroscience
}

\author{
Rie Asano ${ }^{\text {a, }}$, Cedric Boeckx ${ }^{\text {b,c,d }}$, Koji Fujita ${ }^{\mathrm{e}}$

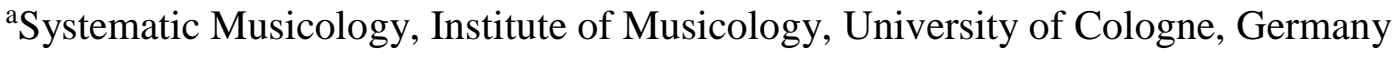 \\ ${ }^{\mathrm{b}}$ Section of General Linguistics, University of Barcelona, Spain \\ ${ }^{c}$ University of Barcelona Institute for Complex Systems (UBICS), Spain \\ ${ }^{\mathrm{d} C a t a l a n}$ Institute for Advanced Studies and Research (ICREA), Spain \\ ${ }^{\mathrm{e}}$ Graduate School of Human and Environmental Studies, Kyoto University, Japan \\ *Corresponding author: rie.asano@uni-koeln.de
}

\begin{abstract}
Comparative research on language, music, and action in cognitive neuroscience keeps finding evidence for both shared and non-shared components of cognitive systems. The discussions, then, tend to quickly fall into the sterile dichotomy between domain-specific vs. domaingeneral options. In this position paper, we take issue with this dichotomy and argue for an alternative account based on neural reuse theories to understand findings on the relationship between language, music, and action. We argue that the differences between those cognitive systems can be explained in terms of the specialization of the same brain mechanism(s) for each domain, which emerges in the course of development and/or evolution.
\end{abstract}

Keywords

Comparative research, neural reuse theories, language, music, action 


\section{Introduction}

Cognitive science has been dominated by intense debates around key notions such as innateness or domain-specificity/modularity. Here we focus on the latter, and based on recent research reviving tensions, we suggest productive ways of going beyond sterile dichotomies. In particular, we capitalize on neural reuse theories and argue that cognitive systems can be understood as different uses of the same mechanisms. Although neural reuse theories have repeatedly gained attention in cognitive neuroscience and provided an explanatory account for the relationship between different cognitive systems (Anderson, 2010; Hernandez et al., 2019; Pulvermüller, 2018), they still often remain completely neglected by current comparative research when discussing issues like domain-specificity vs. domain-generality. So far, comparative research is dominated by classical 'top-down' approaches studying the relationship between language, music, and action based on ready-made theoretical concepts. In contrast, neural reuse theories offer a bottom-up comparative approach investigating different cognitive domains based on a set of basic operations performed by the brain. It is such a bottomup approach that we argue resolves the dichotomy between domain-specific vs. domain-general options. We focus on the relationship between language, music, and action and more specifically their syntactic aspects to illustrate our position, but we think our message is not restricted to these.

2 Sharing and non-sharing: An unresolved issue

Three decades of comparative research on language and music in cognitive neuroscience have adduced evidence for both shared and non-shared components. In the beginning, the explanatory target of comparative research on language and music was the 
discrepancy between findings from neuroimaging on shared neural resources (e.g., Koelsch et al., 2002; Patel, Gibson, Ratner, Besson, \& Holcomb, 1998) and neuropsychology on domainspecificity (e.g., Ayotte, Peretz, \& Hyde, 2002; Peretz et al., 1994). Patel's (2003, 2008) resource-sharing framework resolved this issue in a convincing way by distinguishing shared resources for syntactic integration in the frontal processing region from domain-specific representations in the posterior representation region. The same neural resources were suggested to be engaged in action (e.g., Fitch \& Martins, 2014; Koelsch, 2012), building on early insights from Lashley (1951) and Miller et al. (1960). However, an increasing number of findings on language, music, and action as well as their interactions have revealed that the relationship between these domains is far more complex.

On the one hand, behavioral, neuroimaging, and neuropsychological studies continue to provide evidence for shared neural resources for syntax in language, music, and action. For example, behavioral and electroencephalography studies have shown that language syntactic processing interferes with music syntactic processing (e.g., Fedorenko, Patel, Casasanto, Winawer, \& Gibson, 2009; Koelsch, Gunter, Wittfoth, \& Sammler, 2005; Slevc, Rosenberg, \& Patel, 2009) and action syntactic processing (Casado et al., 2018). Music syntactic knowledge influences action planning in trained pianists (Bianco et al., 2016; Sammler, Novembre, Koelsch, \& Keller, 2013). Functional magnetic resonance imaging (fMRI) studies found that language syntactic processing overlaps with music syntactic processing in Broca's region (Chiang et al., 2018; Kunert, Willems, Casasanto, Patel, \& Hagoort, 2015) and with tool-use in the basal ganglia (Thibault et al., 2021). Agrammatic aphasic patients show deficits in music syntactic processing (Patel, Iversen, Wassenaar, \& Hagoort, 2008) and action planning (Fazio et al., 2009). Children with specific language impairment show the absence of an event-related potential (ERP) component for music syntactic processing (Jentschke, Koelsch, Sallat, \& Friederici, 2008) and abnormal action syntactic processing (Roy et al., 2013). 
These findings can be interpreted in light of the domain-general hierarchical processing mechanism underlying language, music, and action (Fitch \& Martins, 2014).

On the other hand, an ever-increasing number of neuroimaging, meta-analytic, and neuropsychological studies provide evidence in support of functional separability between language, music, and action, both in terms of processing and representation. For example, fMRI studies have revealed a left-lateralized fronto-temporal core language network for 'high-level' linguistic processing including lexical and syntactic processing that is not sensitive to music processing (Chen et al., 2021; Fedorenko, Behr, \& Kanwisher, 2011). Even within Broca's region, there appears to be a portion specialized for language processing (Fedorenko, Duncan, \& Kanwisher, 2012). Moreover, meta-analytic co-activation-based parcellation studies have shown that there are multiple subareas with different functionality even within the left BA44: the anterior portion of the left BA44 is associated with core aspects of language processing and shows connectivity with the left middle temporal gyrus, while action and music are rather located at the posterior portion, which exhibits a different connectivity profile (Clos, Amunts, Laird, Fox, \& Eickhoff, 2013; Papitto, Friederici, \& Zaccarella, 2020). Neuropsychological studies indicate preserved ability for music processing in aphasic patients (Chen et al., 2021; Chiappetta, Patel, \& Thompson, 2022; Slevc, Faroqi-Shah, Saxena, \& Okada, 2016). In sum, arguments have been put forth against the idea of a domain-general hierarchical processing mechanism common to language, music, and action (Fedorenko \& Blank, 2020; Fedorenko \& Shain, 2021; Fedorenko \& Varley, 2016; Zaccarella, Papitto, \& Friederici, 2021).

To some extent, these recently revived claims of neural specializations should not come as a surprise. After all, language, music, and action differ in several obvious ways (items they combine, categories for labeling units, and content they encode / are used to express (Asano \& Boeckx, 2015)). It seems to us that the key question should not be whether music and action show all the features characteristic of language nor whether they activate the core language- 
network. Those questions invariably lead to a contrastive, and ultimately, we think, sterile approach, as we have discussed in detail elsewhere (Asano \& Boeckx, 2015). A more productive question, particularly for the growing number of researchers who have abandoned the classical stance of innately specified cognitive modules, should be how the differences, as well as the similarities, should be understood as emerging in the course of development and/or evolution.

3 Limitations of top-down comparative approaches

Comparative research has already advanced a couple of hypotheses regarding how to understand findings that favor shared and distinctive components of language, music, and action. However, they cannot fully account for the existing data. For example, it has been argued that commonalities between language and music arise as a result of a generic mental process responsible for processing deviant events and have little to do with 'high-level' linguistic processing (Fedorenko \& Varley, 2016). This would be due to the fact that many studies which provided evidence for shared neural resources for language and music syntactic processing used a violation paradigm. However, this explanation does not have universal validity. Syntactically deviant chords in music, but not physically deviant tones (e.g., timbre deviants), influence the left anterior negativity (LAN), an ERP component associated with language syntactic processing (Koelsch et al., 2005). Moreover, there is at least one study providing evidence for shared neural resources for language and music syntactic processing without introducing any violation (Chiang et al., 2018). Thus, there should be something shared between language and music syntactic processing that cannot be explained in terms of a generic mental process for processing deviant events. 
Another possible explanation for domain-commonality is that both domains at issue deal with pitch and rhythm processing and their shared components have little to do with 'highlevel' linguistic processing (Chen et al., 2021). Indeed, prosodic structure parallels musical structure (Heffner \& Slevc, 2015; Lerdahl \& Jackendoff, 1983). However, again, this explanation alone cannot fully account for the existing data. Interference between language and music syntactic processing occurs even though the sentence was visually presented word-byword without any prosodic cues (Carrus, Koelsch, \& Bhattacharya, 2011; Koelsch et al., 2005; Steinbeis \& Koelsch, 2008). Moreover, even though pitch and rhythm processing would partially account for the relationship between language and music, it leaves unexplained how prosody and music relate to syntactic processing in language. Thus, claiming or showing that language and music share general pitch and rhythm processing does not suffice as an explanation for shared components of language and music as seen in their syntactic processing.

Finally, one candidate explanation for the shared properties of language, music, and action is that they draw on domain-general executive function. Language, music, but also action processing, could share resources for working memory (Fitch \& Martins, 2014; Kljajevic, 2010) or cognitive control (Jeon, 2014; Slevc \& Okada, 2015), signaled by activity in Broca's region, especially BA44. Indeed, the majority of studies providing evidence for shared neural resources for language, music, and action syntactic processing makes use of experimental paradigms that impose high control demands on participants (e.g., the need of selecting an alternative interpretation or processing temporally distant elements) (Slevc \& Okada, 2015). Moreover, the executive-function-based explanation can account for hierarchical processing in language, music, and action because control processes to select, maintain, and inhibit goals are hierarchically organized (Asano, Boeckx, \& Seifert, 2021; Fitch \& Martins, 2014; Jeon, 2014; Slevc \& Okada, 2015). Yet, several authors cast doubt on this explanation by pointing to dissociations between language syntactic processing and executive 
function, highlighting the clear separation of the core language network and the domain-general cognitive control network revealed in several studies (Chen et al., 2021; Fedorenko \& Blank, 2020; Fedorenko \& Varley, 2016; Zaccarella et al., 2021). These authors conclude that the shared mechanism between language, music, and action has little to do with language syntactic processing.

So far, comparative research has been devoted to the investigation of whether shared components are 'high-level' linguistic components like syntax or whether they can be explained in terms of executive function. However, such top-down comparative approaches relying on the ready-made concepts of linguistics or psychology are clearly not able to provide a comprehensive explanation for the complex relationship between language, music, and action. Moreover, caution is needed when we map theoretical concepts from linguistics or psychology onto neuroscientific concepts (for discussions, see, e.g., Embick \& Poeppel, 2015; Lenartowicz, Kalar, Congdon, \& Poldrack, 2010; Poeppel \& Embick, 2005; Price \& Friston, 2005). Although the labeling of cognitive phenomena with top-down concepts is a reasonable first approximation, it could eventually obscure the need for detailed mechanistic investigations (Brick, Hood, Ekroll, \& De-Wit, 2022). In what follows, based on the neural reuse theories (Anderson, 2010), we propose an alternative account to these top-down approaches in order to resolve the shared vs. distinct dichotomy in comparative research on language, music, and action.

4 A perspective from neural reuse theories: Going beyond the specific/general dichotomy

4.1 Neural reuse theories: Perspectives from bottom-up

Neural reuse theories come in many guises: massive redeployment hypothesis (Anderson, 2007), neuronal recycling hypothesis (Dehaene \& Cohen, 2007), or neuronal 
retuning hypothesis (Matchin, 2018). However, all of them revolve around the central idea that brain regions or circuits established for one function can be reused for various new functions during evolution (i.e., exaptation) or development, often without losing their initial functions (for reviews, see Anderson, 2010; Hernandez et al., 2019). For example, comprehending action verbs "kick", "pick", and "lick" activates motor regions associated with leg, arm, and face movements (Pulvermüller, 2005), indicating that those regions are reused in a newer context of language comprehension (Anderson, 2010). Moreover, as non-human primates display a certain degree of hierarchical combinatorial ability in the domain of action (Byrne \& Russon, 1998; Hayashi, 2007; Matsuzawa, 1991), its reuse in syntax of language and music was suggested (Asano, 2021; Fujita, 2016; Greenfield, 1991; Hilton, Asano, \& Boeckx, 2021; Pulvermüller, 2014). They all argue for continuity from action to language and music.

Moreover, all neural reuse theories agree on the distinction between the notion of “working”, i.e., a canonical information processing operation performed by a region or local anatomical circuits, and the notion of "use", i.e., a cognitive function to which the working is applied in a unique way (Anderson, 2010). Thus, different cognitive functions such as language, music, and action could be explained in terms of different uses of the same working. Differences between those cognitive functions in terms of neural segregation can be explained in terms of the domain-specific configuration of the networks in which a region featuring a common operation is embedded. Importantly, neural reuse theories are in line with network approaches that suggest that computations performed by the language network (and its subregions) are unique and do not parallel that of music and action (Chen et al., 2021; Fedorenko \& Blank, 2020; Fedorenko \& Thompson-Schill, 2014). Neural reuse theories constitute a variant of the network approach, putting more emphasis on the common working of a region used in different domain-specific networks. 
Neural reuse theories offer a bottom-up perspective to comparative research on language, music, and action by studying different cognitive domains based on a set of basic operations performed by the brain. They aim to identify the underlying mechanisms of cognitive systems independent of top-down linguistic or psychological concepts and to do so in a more neuro-biologically plausible way (Anderson, 2015; Pulvermüller, 2010, 2018; Stocco, 2019). Here, "mechanism" is understood as the way how different constituent parts performing unique operations work in tandem to bring about a given phenomenon (Bechtel \& Shagrir, 2015). Moreover, neural reuse theories introduce developmental and evolutionary perspectives to comparative research on language, music, and action, complementing the studies on the fully developed brain in cognitive neuroscience (Anderson, 2010, 2016; Dekker \& Karmiloff-Smith, 2010; Hernandez et al., 2019). Thus, neural reuse theories explain the relationship between language, music, and action in terms of common basic mechanisms that get organized into specialized networks in the course of development and/or evolution. Accordingly, there is no such dichotomy as domain-general hubs vs. domain-specific networks of the sort that can be found in the literature (e.g., in Figure 1 of Fedorenko \& Shain, 2021).

Bottom-up perspectives of neural reuse theories have another advantage: they facilitate research on the relationship between syntax in language, music, and action beyond the cortex. For example, the significant role of the basal ganglia in language syntactic processing is repeatedly emphasized (Birba et al., 2017; Dominey \& Inui, 2009; Kotz, Schwartze, \& Schmidt-Kassow, 2009; Lieberman, 2002, 2016; Ullman, 2006), but did not get enough attention in comparative research on syntax in language, music, and action (but see Asano et al., 2021). The same can be said concerning the cerebellum, the hippocampus, the thalamus, and perhaps additional regions as well (Adamaszek \& Kirkby, 2016; Boeckx \& Theofanopoulou, 2018; Duff \& Brown-Schmid, 2017). The traditional corticocentric view that 'higher' cognitive functions (e.g., 'syntax') are implemented by cortical structures and 'lower 
functions' by subcortical structures retains its dominant position despite the evidence pointing to cortical and subcortical structures working in tandem to bring about 'higher' cognitive functions (DeCasien, Barton, \& Higham, 2022; Parvizi, 2009). Neural reuse theories overcome such a high- vs. low-level dichotomy by arguing that putatively low-level neural circuits are used and reused by high-level cognitive domains (Anderson, 2010). That is, there is no clearcut distinction between neural circuits underlying high- and low-level processes. This is in line with the approach regarding motor and cognitive processes more as a gradient rather than as a categorical division (Marvel, Morgan, \& Kronemer, 2019). Ultimately, it is also in agreement with the approach advocated by Cisek (2019) on "phylogenetic refinement" and several contributions to the thematic issue "Systems neuroscience through the lens of evolutionary theory" (see Cisek \& Hayden, 2022 for an overview of the contributions).

4.2 Application to comparative research on language, music, and action

For comparative research on language, music, and action, one candidate working of the prefrontal cortex is the active maintenance of representations as evidenced by sustained neural activity (Fuster, 2008; E. K. Miller \& Buschman, 2008; E. K. Miller \& Cohen, 2001), which is also a possible working of BA44 and is crucial for language, music, and action to establish structural relationships between elements (Asano et al., 2021; Fitch \& Martins, 2014). For example, the language-selective part of the left BA44 shows significant activation in challenging verbal working memory tasks (Fedorenko et al., 2012). Even the languageselective part of the left BA45 is devoted to the maintenance of the representations of language retrieved from long-term memory (Matchin, 2018). Thus, it is possible that there are multiple specialized storage and retrieval mechanisms within Broca's region (Fitch, 2018). According to neural reuse theories, specialized subparts of BA44 for language, music, and action with different connectivity profiles (Clos et al., 2013; Papitto et al., 2020) can be understood in such 
a way that the common maintenance operation is embedded in different networks to solve different computational problems.

Moreover, the frontal cortex is organized along an abstraction gradient from the posterior portion buffering the goals associated with the concrete motor act (e.g., grabbing a pot) to the anterior portion maintaining abstract, temporally extended goals (e.g., coffee making) (Badre, 2008). Language processing is also organized alongside the abstraction gradient from phonology in the more posterior region to syntax to semantics in the more anterior region (Uddén \& Bahlmann, 2012). In music, too, abstract structural processing is associated with a more anterior region than the one devoted to motor processing (Bianco et al., 2021). In processing sentences and musical sequences with center-embedding, the relationship between temporally distant elements should be established (Jeon, 2014). However, a review of neuroimaging studies on syntactic processing in the two domains showed that music syntactic processing relies on the coordination of abstract and motor processing circuits, while language syntactic processing more preferably engages abstract processing circuits (Asano et al., 2021). Thus, the difference between language, music, and action within the left BA44 as seen in some meta-analyses (Clos et al., 2013; Papitto et al., 2020) could also be explained in terms of this abstraction gradient with "language" clustering at the anterior portion and "music" and "action" clustering around the posterior portion.

It is not just the frontal maintenance operation that builds the common basis for language, music, and action, but also the inhibition and selection operation of the basal ganglia to control cortically maintained representations via the coordinated activity of the direct and indirect pathways (Lieberman, 2002; Marsden \& Obeso, 1994). The basal ganglia are interconnected with the frontal cortex and form functionally segregated cortico-basal gangliathalamocortical (CBGT) circuits reflecting the gradient organization of the frontal cortex $(\mathrm{G}$. E. Alexander, Delong, \& Strick, 1986; Haber, 2003). The basal ganglia operations together 
with the frontal maintenance operation constitute the hierarchical control mechanism underlying not only language, music, and action, but also cognitive control and working memory (Asano et al., 2021; Badre \& Nee, 2018; Lustig, Matell, \& Meck, 2005). Processing sentences, musical sequences, complex actions (e.g., coffee making), and cognitive control or working memory tasks (e.g., Wisconsin Card Sorting Task) all require a balanced choice between multiple representations. From the bottom-up perspective of neural reuse theories, these cognitive phenomena are not rigid categories, but rather, different uses of the same mechanism.

Importantly, such a mechanistic approach ideally goes hand in hand with a computational approach. For example, the Hierarchical Reinforcement Learning model is a computational neurocognitive model implementing the abstraction gradient as well as the maintenance, inhibition, and selection operations of the CBGT circuits and successfully simulates the behavior and neural dynamics of some cognitive control tasks (Badre \& Frank, 2012; Frank \& Badre, 2012). The Hierarchical reinforcement learning system adaptively learns to represent and maintain information at different levels of abstraction to optimally perform the task. The Hierarchical Error Representation model also implements the operations of the CBGT circuits and integrates them with a prediction mechanism (W. H. Alexander \& Brown, 2018). The application of such models to comparative research on language, music, and action facilitates the exploration of how different domains emerge from common mechanisms. Simulation works with biologically plausible neurocomputational models can provide a proofof-concept that the same neural circuits can realize the mechanistic basis of diverse cognitive domains (Pulvermüller, 2018).

Needless to say, what we discussed here is only a small subpart of the common mechanisms underlying language, music, and action. For example, a detailed analysis of the parietal and temporal cortex in light of neural reuse theories is likely to provide additional 
insights. The posterior temporal cortex, especially the posterior middle temporal gyrus, is known as another important region involved in language syntactic processing to decode auditory sequences into hierarchical structures and link these with conceptual structures (Matchin \& Hickok, 2020; Pylkkänen, 2019). The angular gyrus, the anterior superior temporal sulcus, and the temporal pole are sensitive to the constituent size of meaningful phrases (Pallier, Devauchelle, \& Dehaene, 2011). For music, the generation of hierarchical structures according to a recursive rule activates the posterior superior temporal gyrus (Martins et al., 2020). The fronto-parietal and fronto-temporal networks are involved in pianists' motor planning and music syntactic processing, respectively (Bianco et al., 2021). For action, tool-use planning engages the parietal and temporal lobe in addition to the frontal lobe, which are interconnected to form fronto-parieto-temporal networks (for a review, see Maravita \& Romano, 2018).

Although more research is necessary to identify the working of the parietal and temporal cortex, there are leading hypotheses such as the encoding of abstract structural relations (or rules) for the parietal lobe (Orpella et al., 2020; Summerfield, Luyckx, \& Sheahan, 2020) as well as hierarchical combinatorial object formation for the temporal lobe (Rauschecker \& Scott, 2009). The notion of an abstraction gradient is also applicable to the parietal and the temporal cortex (Braunsdorf et al., 2021; Choi, Drayna, \& Badre, 2018; Fuster, 2008). Clarification of the operations performed by the parietal and temporal cortex could further inform the relationship between language, music, and action in light of neural reuse theories.

\subsection{Towards evolutionary-developmental approaches}

From the perspective of neural reuse theories, we should expect specialization to emerge in the course of development. For instance, the left inferior frontal gyrus as well as the left posterior superior temporal gyrus of the core language network is expected to become functionally specialized as their structural connection via the arcuate fasciculus matures in the 
course of the development (Skeide \& Friederici, 2016). Strikingly, a study conducted with an individual born without the left temporal lobe showed that the language-selective frontal areas do not emerge without the ipsilateral temporal lobe (Tuckute et al., 2022). The same study also showed that the non-language-dominant right hemispheric fronto-temporal network is sufficient for language function, indicating that the right frontal and temporal regions implement the same operations as their left counterparts and can become specialized for performing linguistic computation. The right inferior frontal gyrus is generally more associated with music, action execution and inhibition, social cognition, emotion, and executive function (Hartwigsen, Neef, Camilleri, Margulies, \& Eickhoff, 2019). Thus, the specialized languagenetwork is an emergent property of the developing brain.

Once one takes the specialized core language network as an emerging property of the cortical as well as subcortical (re-)organization, and language, music, and action syntactic processing as different uses of the same mechanism(s), the boundaries between domainspecific and domain-general processes blur and a novel, more productive comparative perspective emerges. From the perspective of the neural reuse theories, research on the developing brain becomes more central to comparative research on language, music, and action (see also Goswami, 2012; Maffongelli, D’Ausilio, Fadiga, \& Daum, 2019). In particular, the neuroconstructivist approach to brain development (Dekker \& Karmiloff-Smith, 2011; Karmiloff-Smith, 2013) provides some useful predictions. According to this approach, the brain's initial state is coarsely coded so that each neurobiological bias (e.g., cell types, firing thresholds) is more suitable for certain types of information processing over others. Through neural competition, the coarsely coded system becomes increasingly more domain-specific over time in the development and gives rise to the developed brain of the adults with computational specializations. The neuroconstructivist approach predicts that the infant's 
developing brain should show more broad activations than the specialized adult's brain to perform specific computations (Karmiloff-Smith, 2013).

We think that this point is important because so far, studies that highlight the existence of a specialized core language network mainly focus on the first-language processing of adults, with a fully developed brain in which language processing is automatized. However, language processing in the subjects with a low level of proficiency such as children and adult second language learners is known to engage far more extended brain regions than for adult first language speakers (Jeon \& Friederici, 2015). Thus, it is possible that language processing in the (developing) brain without automatized processing relies on the resources of broader brain regions than the developed brain with automatized processing does. For example, studies conducted with German 2-year-olds showed that the early left anterior negativity (ELAN) associated with automatic language syntactic processing emerges later in the development, while the P600 associated with controlled syntactic processing is present earlier (Oberecker \& Friederici, 2006; Oberecker, Friedrich, \& Friederici, 2005). This points to a developmental change toward more automatic syntactic processing (Friederici, 2017). P600 is affected by the lesions to the frontal, temporal, and/or parietal sites (Kaan, 2007) as well as the deficient basal ganglia (Friederici \& Kotz, 2003) and might be indicative of more widespread brain activations.

The neuroconstructivist approach to brain development together with the mechanistic approach accompanied by computational neurocognitive modeling could advance future research on neural specialization. For example, the Hierarchical Reinforcement Learning model can be regarded as a model of the neural specialization where control processes get specialized via the learning mechanism implemented in cortico-basal ganglia-thalamocortical circuits. The interplay between the frontal cortex and the basal ganglia plays a central role in the acquisition of abstract rules through statistical learning (E. K. Miller \& Buschman, 2008; Orpella, Mas-Herrero, Ripollés, Marco-Pallarés, \& de Diego-Balaguer, 2021; Ullman, Earle, 
Walenski, \& Janacsek, 2020). Especially, the basal ganglia are considered to implement an implicit, associative learning mechanism and tune the cortico-cortical top-down control pathways (E. K. Miller \& Buschman, 2008; Ullman et al., 2020). This is in line with the findings that the early learning mode as seen in infants (or children) is associative, implicit, and independent of top-down control, while the adult learning mode relies more on top-down control and explicit knowledge (Friederici, Mueller, Sehm, \& Ragert, 2013; Smalle, Daikoku, Szmalec, Duyck, \& Möttönen, 2022; Ullman et al., 2020). Thus, in light of neural reuse theories, research on development and learning can inform computational research focusing on neurocognitive mechanisms and vice versa.

Last but not least, neural reuse theories have important implications for evolutionary research, as they point to a more continuous view of the evolution of cognitive functions in a similar vein to Darwin's idea of descent with modification (see also Boeckx \& Fujita, 2014). For example, Anderson's massive redeployment hypothesis investigates the evolution of the brain in light of an exaptive account of cognitive functions by suggesting that a new cognitive function can emerge based on the recombination of the old, existing components (Anderson, 2007). Another related idea is "duplication and divergence" by which new systems are created based on modified copies of older systems (Chakraborty \& Jarvis, 2015; Marcus, 2006; Marcus, Rabaglia, \& Rabagliati, 2006), as argued for the evolution of vocal learning through the duplication of the brain mechanisms from an ancient motor learning (Feenders et al., 2008). Importantly, neural reuse theories imply the emergence of different cognitive systems through the modification of shared components. Thus, again, it is unsurprising that language, music, and action show specializations of brain networks. However, as we argue here, the existence of these specialized networks does not reject the existence of shared mechanisms that serve as common bases for the emerging specializations of cognitive systems. Neural reuse theories make it clear that cognitive systems such as language, music, and action cannot be explained 
in terms of the domain-specific vs. domain-general dichotomy. Rather, they are better understood as different uses of the same mechanisms.

\section{Concluding remarks}

To summarize, neural reuse theories suggest that syntactic aspects of language, music, and action are emergent properties of the brain, arising through the specialization of the common brain mechanisms in the course of development and/or evolution. As the brain becomes specialized for each domain over time, likely through automatization and the accumulation of sub-routines, the less language, music, and action syntactic processing should rely on the shared neural resources, except when there is an unusually large task demand. In the developed, adult brain, solving specific computational problems should result in more segregated, domain-specific networks than in the developing brain. This, we insist, should not come as a surprise. Importantly, the operations of the regions should remain unchanged even though it is used to solve different computational problems in specialized networks. Thus, even though differences between language, music, and action can be detected in neuroimaging studies, comparative research should take neural reuse theories into account and avoid the sterile dichotomy between the domain-specific vs. domain-general options. Especially now that the idea of innate modules has lost its appeal, the question driving research across cognitive domains should not be "whether there are domain-specific and -general brain regions / networks", but rather "how and why the brain becomes specialized as it does, when it does". 
Acknowledgments

We would like to thank the editor, two anonymous reviewers, and Ani Patel for their helpful comments on earlier versions of our manuscript.

Funding: This work was supported by MEXT/JSPS Grant-in-Aid for Scientific Research on Innovative Areas \#4903 (Evolinguistics) [grant number JP17H06379] and the Spanish Ministry of Science and Innovation [grant number PID2019-107042GB-I00].

Declarations of interest: none

\section{References}

Adamaszek, M., \& Kirkby, K. C. (2016). Cerebellum and grammar processing. In P. Mariën \& M. Manto (Eds.), The linguistic cerebellum (pp. 81-105). Amsterdam: Elsevier. https://doi.org/10.1016/B978-0-12-801608-4.00005-0

Alexander, G. E., Delong, M. R., \& Strick, P. L. (1986). Parallel organization of functionally segregated circuits linking basal ganglia and cortex. Annual Review of Neuroscience, 9(1), 357-381. https://doi.org/10.1146/annurev.ne.09.030186.002041

Alexander, W. H., \& Brown, J. W. (2018). Frontal cortex function as derived from hierarchical predictive coding. Scientific Reports, 8(1), 1-11. https://doi.org/10.1038/s41598-01821407-9

Anderson, M. L. (2007). Evolution of cognitive function via redeployment of brain areas. The Neuroscientist, 13(1), 13-21. https://doi.org/10.1177/1073858406294706

Anderson, M. L. (2010). Neural reuse: A fundamental organizational principle of the brain. Behavioral and Brain Sciences, 33(4), 245-266. 
https://doi.org/10.1017/S0140525X10000853

Anderson, M. L. (2015). Mining the brain for a new taxonomy of the mind. Philosophy Compass, 10(1), 68-77. https://doi.org/10.1111/phc3.12155

Anderson, M. L. (2016). Neural reuse in the organization and development of the brain. Developmental Medicine \& Child Neurology, 58, 3-6. https://doi.org/10.1111/dmcn.13039

Asano, R. (2021). The evolution of hierarchical structure building capacity for language and music: A bottom-up perspective. Primates. https://doi.org/10.1007/s10329-021-00905-x

Asano, R., \& Boeckx, C. (2015). Syntax in language and music: What is the right level of comparison? Frontiers in Psychology, 6, 942. https://doi.org/10.3389/fpsyg.2015.00942

Asano, R., Boeckx, C., \& Seifert, U. (2021). Hierarchical control as a shared neurocognitive mechanism for language and music. Cognition, 216, 104847. https://doi.org/10.1016/j.cognition.2021.104847

Ayotte, J., Peretz, I., \& Hyde, K. (2002). Congenital amusia. A group study of adults afflicted with a music-specific disorder. Brain, 125(2), 238-251. https://doi.org/10.1093/brain/awf028

Badre, D. (2008). Cognitive control, hierarchy, and the rostro-caudal organization of the frontal lobes. Trends in Cognitive Sciences, 12(5), 193-200. https://doi.org/10.1016/j.tics.2008.02.004

Badre, D., \& Frank, M. J. (2012). Mechanisms of hierarchical reinforcement learning in cortico-striatal circuits 2: Evidence from fMRI. Cerebral Cortex, 22(3), 527-536. https://doi.org/10.1093/cercor/bhr117

Badre, D., \& Nee, D. E. (2018). Frontal cortex and the hierarchical control of behavior. Trends in Cognitive Sciences, 22(2), 170-188. https://doi.org/10.1016/j.tics.2017.11.005

Bechtel, W., \& Shagrir, O. (2015). The non-redundant contributions of Marr's three levels of 
analysis for explaining information-processing mechanisms. Topics in Cognitive Science, 7(2), 312-322. https://doi.org/10.1111/tops.12141

Bianco, R., Novembre, G., Keller, P. E., Scharf, F., Friederici, A. D., Villringer, A., \& Sammler, D. (2016). Syntax in action has priority over movement selection in piano playing: An ERP study. Journal of Cognitive Neuroscience, 28(1), 41-54. https://doi.org/10.1162/jocn_a_00873

Bianco, R., Novembre, G., Ringer, H., Kohler, N., Keller, P. E., Villringer, A., \& Sammler, D. (2021). Lateral prefrontal cortex is a hub for music production from structural rules to movements. Cerebral Cortex. https://doi.org/10.1093/cercor/bhab454

Birba, A., García-Cordero, I., Kozono, G., Legaz, A., Ibáñez, A., Sedeño, L., \& García, A. M. (2017). Losing ground: Frontostriatal atrophy disrupts language embodiment in Parkinson's and Huntington's disease. Neuroscience \& Biobehavioral Reviews, 80, 673687. https://doi.org/10.1016/j.neubiorev.2017.07.011

Boeckx, C., \& Fujita, K. (2014). Syntax, action, comparative cognitive science, and Darwinian thinking. Frontiers in Psychology, 5, 627. https://doi.org/10.3389/fpsyg.2014.00627

Boeckx, C., \& Theofanopoulou, C. (2018). (Neural) Syntax. In Á. J. Gallego \& R. Martin (Eds.), Language, syntax, and the natural sciences (pp. 295-315). Cambridge: Cambridge University Press. https://doi.org/10.1017/9781316591529.016

Braunsdorf, M., Blazquez Freches, G., Roumazeilles, L., Eichert, N., Schurz, M., Uithol, S., ... Mars, R. B. (2021). Does the temporal cortex make us human? A review of structural and functional diversity of the primate temporal lobe. Neuroscience \& Biobehavioral Reviews, 131, 400-410. https://doi.org/10.1016/j.neubiorev.2021.08.032

Brick, C., Hood, B., Ekroll, V., \& De-Wit, L. (2022). Illusory essences: A bias holding back theorizing in psychological science. Perspectives on Psychological Science, 17(2), 491506. https://doi.org/10.1177/1745691621991838 
Byrne, R. W., \& Russon, A. E. (1998). Learning by imitation: A hierarchical approach. Behavioral and Brain $\quad$ Sciences, 667-684. https://doi.org/10.1017/S0140525X98001745

Carrus, E., Koelsch, S., \& Bhattacharya, J. (2011). Shadows of music-language interaction on low frequency brain oscillatory patterns. Brain and Language, 119(1), 50-57. https://doi.org/10.1016/j.bandl.2011.05.009

Casado, P., Martín-Loeches, M., León, I., Hernández-Gutiérrez, D., Espuny, J., Muñoz, F., ... de Vega, M. (2018). When syntax meets action: Brain potential evidence of overlapping between language and motor sequencing. Cortex, 100, 40-51. https://doi.org/10.1016/j.cortex.2017.11.002

Chakraborty, M., \& Jarvis, E. D. (2015). Brain evolution by brain pathway duplication. Philosophical Transactions of the Royal Society B: Biological Sciences, 370(1684), 20150056. https://doi.org/10.1098/rstb.2015.0056

Chen, X., Affourtit, J., Ryskin, R., Regev, T. I., Norman-Haignere, S., Jouravlev, O., ... Fedorenko, E. (2021). The human language system does not support music processing. BioRxiv. https://doi.org/10.1101/2021.06.01.446439

Chiang, J. N., Rosenberg, M. H., Bufford, C. A., Stephens, D., Lysy, A., \& Monti, M. M. (2018). The language of music: Common neural codes for structured sequences in music and natural language. Brain and Language, 185, 30-37. https://doi.org/10.1016/j.bandl.2018.07.003

Chiappetta, B., Patel, A. D., \& Thompson, C. K. (2022). Musical and linguistic syntactic processing in agrammatic aphasia: An ERP study. Journal of Neurolinguistics, 62, 101043. https://doi.org/10.1016/j.jneuroling.2021.101043

Choi, E. Y., Drayna, G. K., \& Badre, D. (2018). Evidence for a functional hierarchy of association networks. Journal of Cognitive Neuroscience, 30(5), 722-736. 
https://doi.org/10.1162/jocn_a_01229

Cisek, P. (2019). Resynthesizing behavior through phylogenetic refinement. Attention, Perception, \& Psychophysics, 81(7), 2265-2287. https://doi.org/10.3758/s13414-01901760-1

Cisek, P., \& Hayden, B. Y. (2022). Neuroscience needs evolution. Philosophical Transactions of the Royal Society B: Biological Sciences, 377(1844), 20200518. https://doi.org/10.1098/rstb.2020.0518

Clos, M., Amunts, K., Laird, A. R., Fox, P. T., \& Eickhoff, S. B. (2013). Tackling the multifunctional nature of Broca's region meta-analytically: Co-activation-based parcellation of area 44. NeuroImage, 83, 174-188. https://doi.org/10.1016/j.neuroimage.2013.06.041

DeCasien, A. R., Barton, R. A., \& Higham, J. P. (2022). Understanding the human brain: Insights from comparative biology. Trends in Cognitive Sciences, 1-14. https://doi.org/10.1016/j.tics.2022.02.003

Dehaene, S., \& Cohen, L. (2007). Cultural recycling of cortical maps. Neuron, 56(2), 384-398. https://doi.org/10.1016/j.neuron.2007.10.004

Dekker, T. M., \& Karmiloff-Smith, A. (2010). The importance of ontogenetic change in typical and atypical development. Behavioral and Brain Sciences, 33(4), 271-272. https://doi.org/10.1017/S0140525X10001019

Dekker, T. M., \& Karmiloff-Smith, A. (2011). The dynamics of ontogeny: A neuroconstructivist perspective on genes, brains, cognition and behavior. In Progress in Brain Research (Vol. 189, pp. 23-33). https://doi.org/10.1016/B978-0-444-53884$0.00016-6$

Dominey, P. F., \& Inui, T. (2009). Cortico-striatal function in sentence comprehension: Insights from neurophysiology and modeling. Cortex, 45(8), 1012-1018. 
https://doi.org/10.1016/j.cortex.2009.03.007

Duff, M. C., \& Brown-Schmid, S. (2017). Hippocampal contributions to language use and processing. In D. E. Hannula \& M. C. Duff (Eds.), The hippocampus from cells to systems: Structure, connectivity, and functional contributions to memory and flexible cognition (pp. 503-536). Cham: Springer. https://doi.org/10.1007/978-3-319-50406-3

Embick, D., \& Poeppel, D. (2015). Towards a computational(ist) neurobiology of language: Correlational, integrated and explanatory neurolinguistics. Language, Cognition and Neuroscience, 30(4), 357-366. https://doi.org/10.1080/23273798.2014.980750

Fazio, P., Cantagallo, A., Craighero, L., D’ausilio, A., Roy, A. C., Pozzo, T., ... Fadiga, L. (2009). Encoding of human action in Broca's area. Brain, 132, 1980-1988. https://doi.org/10.1093/brain/awp118

Fedorenko, E., Behr, M. K., \& Kanwisher, N. (2011). Functional specificity for high-level linguistic processing in the human brain. Proceedings of the National Academy of Sciences, 108(39), 16428-16433. https://doi.org/10.1073/pnas.1112937108

Fedorenko, E., \& Blank, I. A. (2020). Broca's area is not a natural kind. Trends in Cognitive Sciences, 24(4), 270-284. https://doi.org/10.1016/j.tics.2020.01.001

Fedorenko, E., Duncan, J., \& Kanwisher, N. (2012). Language-Selective and domain-general regions lie side by side within Broca's area. Current Biology, 22(21), 2059-2062. https://doi.org/10.1016/j.cub.2012.09.011

Fedorenko, E., Patel, A. D., Casasanto, D., Winawer, J., \& Gibson, E. (2009). Structural integration in language and music: Evidence for a shared system. Memory \& Cognition, 37(1), 1-9. https://doi.org/10.3758/MC.37.1.1

Fedorenko, E., \& Shain, C. (2021). Similarity of computations across domains does not imply shared implementation: The case of language comprehension. Current Directions in Psychological Science, 30(6), 526-534. https://doi.org/10.1177/09637214211046955 
Fedorenko, E., \& Thompson-Schill, S. L. (2014). Reworking the language network. Trends in Cognitive Sciences, 18(3), 120-126. https://doi.org/10.1016/j.tics.2013.12.006

Fedorenko, E., \& Varley, R. (2016). Language and thought are not the same thing: Evidence from neuroimaging and neurological patients. Annals of the New York Academy of Sciences, 1369(1), 132-153. https://doi.org/10.1111/nyas.13046

Feenders, G., Liedvogel, M., Rivas, M., Zapka, M., Horita, H., Hara, E., ... Jarvis, E. D. (2008). Molecular mapping of movement-associated areas in the avian brain: A motor theory for vocal learning origin. PLoS ONE, 3(3), e1768. https://doi.org/10.1371/journal.pone.0001768

Fitch, W. T. (2018). What animals can teach us about human language: The phonological continuity hypothesis. Current Opinion in Behavioral Sciences, 21, 68-75. https://doi.org/10.1016/j.cobeha.2018.01.014

Fitch, W. T., \& Martins, M. D. (2014). Hierarchical processing in music, language, and action: Lashley revisited. Annals of the New York Academy of Sciences, 1316(1), 87-104. https://doi.org/10.1111/nyas. 12406

Frank, M. J., \& Badre, D. (2012). Mechanisms of hierarchical reinforcement learning in corticostriatal circuits 1: Computational analysis. Cerebral Cortex, 22(3), 509-526. https://doi.org/10.1093/cercor/bhr114

Friederici, A. D. (2017). Language in our brain: The origins of a uniquely human capacity. Cambridge, MA: The MIT Press.

Friederici, A. D., \& Kotz, S. A. (2003). The brain basis of syntactic processes: Functional imaging and lesion studies. NeuroImage, 20(SUPPL. 1), S8-S17. https://doi.org/10.1016/j.neuroimage.2003.09.003

Friederici, A. D., Mueller, J. L., Sehm, B., \& Ragert, P. (2013). Language learning without control: The role of the PFC. Journal of Cognitive Neuroscience, 25(5), 814-821. 
https://doi.org/10.1162/jocn_a_00350

Fujita, K. (2016). On the parallel evolution of syntax and lexicon: A Merge-only view. Journal of Neurolinguistics, 1-15. https://doi.org/10.1016/j.jneuroling.2016.05.001

Fuster, J. M. (2008). The prefrontal cortex. Amsterdam: Elsevier Academic Press.

Goswami, U. (2012). Language, music, and children's brains: A rhythmic timing perspective on language and music as cognitive systems. In P. Rebuschat, M. Rohrmeier, J. A. Hawkins, \& I. Cross (Eds.), Language and music as cognitive systems (pp. 292-301). Oxford, New York: Oxford University Press. https://doi.org/10.1093/acprof

Greenfield, P. M. (1991). Language, tools and brain: The ontogeny and phylogeny of hierarchically organized sequential behavior. Behavioral and Brain Sciences, 14, 531595. https://doi.org/10.1017/S0140525X00071235

Haber, S. N. (2003). The primate basal ganglia: Parallel and integrative networks. Journal of Chemical Neuroanatomy, 26(4), $317-330$. https://doi.org/10.1016/j.jchemneu.2003.10.003

Hartwigsen, G., Neef, N. E., Camilleri, J. A., Margulies, D. S., \& Eickhoff, S. B. (2019). Functional segregation of the right inferior frontal gyrus: Evidence from coactivationbased parcellation. Cerebral Cortex, 29(4), 1532-1546. https://doi.org/10.1093/cercor/bhy049

Hayashi, M. (2007). A new notation system of object manipulation in the nesting-cup task for chimpanzees and humans. Cortex, 43(3), 308-318. https://doi.org/10.1016/S00109452(08)70457-X

Heffner, C. C., \& Slevc, L. R. (2015). Prosodic structure as a parallel to musical structure. Frontiers in Psychology, 6, 1962. https://doi.org/10.3389/fpsyg.2015.01962

Hernandez, A. E., Claussenius-Kalman, H. L., Ronderos, J., Castilla-Earls, A. P., Sun, L., Weiss, S. D., \& Young, D. R. (2019). Neuroemergentism: A framework for studying 
cognition and the brain. Journal of Neurolinguistics, 49, 214-223. https://doi.org/10.1016/j.jneuroling.2017.12.010

Hilton, C. B., Asano, R., \& Boeckx, C. (2021). Why musical hierarchies? Behavioral and Brain Sciences, 44, e100. https://doi.org/10.1017/S0140525X20001338

Jentschke, S., Koelsch, S., Sallat, S., \& Friederici, A. D. (2008). Children with specific language impairment also show impairment of music-syntactic processing. Journal of Cognitive Neuroscience, 20(11), 1940-1951. https://doi.org/10.1162/jocn.2008.20135

Jeon, H.-A. (2014). Hierarchical processing in the prefrontal cortex in a variety of cognitive domains. Frontiers in Systems Neuroscience, 223. https://doi.org/10.3389/fnsys.2014.00223

Jeon, H.-A., \& Friederici, A. D. (2015). Degree of automaticity and the prefrontal cortex. Trends in Cognitive Sciences, 19(5), 244-250. https://doi.org/10.1016/j.tics.2015.03.003

Kaan, E. (2007). Event-related potentials and language processing: A brief overview. Language and Linguistics Compass, 1(6), 571-591. https://doi.org/10.1111/j.1749818X.2007.00037.x

Karmiloff-Smith, A. (2013). Challenging the use of adult neuropsychological models for explaining neurodevelopmental disorders: Developed versus developing brains. Quarterly Journal of Experimental Psychology, 66(1), 1-14. https://doi.org/10.1080/17470218.2012.744424

Kljajevic, V. (2010). Is syntactic working memory language specific? Psihologija, 43(1), 85101. https://doi.org/10.2298/PSI1001085K

Koelsch, S. (2012). Brain and music. Oxford: Wiley-Blackwell.

Koelsch, S., Gunter, T. C., v. Cramon, D. Y., Zysset, S., Lohmann, G., \& Friederici, A. D. (2002). Bach speaks: A cortical "language-network" serves the processing of music. NeuroImage, 17(2), 956-966. https://doi.org/10.1006/nimg.2002.1154 
Koelsch, S., Gunter, T. C., Wittfoth, M., \& Sammler, D. (2005). Interaction between syntax processing in language and in music: An ERP Study. Journal of Cognitive Neuroscience, 17(10), 1565-1577. https://doi.org/10.1162/089892905774597290

Kotz, S. A., Schwartze, M., \& Schmidt-Kassow, M. (2009). Non-motor basal ganglia functions: A review and proposal for a model of sensory predictability in auditory language $\quad$ perception. $\quad$ Cortex, $\quad 45(8), \quad 982-990$. https://doi.org/10.1016/j.cortex.2009.02.010

Kunert, R., Willems, R. M., Casasanto, D., Patel, A. D., \& Hagoort, P. (2015). Music and language syntax interact in Broca's area: An fMRI study. PLoS ONE, 10(11), 1-16. https://doi.org/10.1371/journal.pone.0141069

Lashley, K. (1951). The problem of serial order in behavior. In L. A. Jeffress (Ed.), Cerebral mechanisms in behavior: The Hixon symposium (pp. 112-147). New York: Wiley.

Lenartowicz, A., Kalar, D. J., Congdon, E., \& Poldrack, R. A. (2010). Towards an ontology of cognitive control. Topics in Cognitive Science, 2(4), 678-692. https://doi.org/10.1111/j.1756-8765.2010.01100.x

Lerdahl, F., \& Jackendoff, R. (1983). A generative theory of tonal music. Cambridge, MA: MIT Press.

Lieberman, P. (2002). On the nature and evolution of the neural bases of human language. American Journal of Physical Anthropology, 119(S35), 36-62. https://doi.org/10.1002/ajpa.10171

Lieberman, P. (2016). The evolution of language and thought. Journal of Anthropological Sciences, 94, 1-20. https://doi.org/10.4436/jass.94029

Lustig, C., Matell, M. S., \& Meck, W. H. (2005). Not “just” a coincidence: Frontal-striatal interactions in working memory and interval timing. Memory (Hove, England), 13(3-4), 441-448. https://doi.org/10.1080/09658210344000404 
Maffongelli, L., D’Ausilio, A., Fadiga, L., \& Daum, M. M. (2019). The ontogenesis of action syntax. Collabra: Psychology, 5(1), 1-11. https://doi.org/10.1525/collabra.215

Maravita, A., \& Romano, D. (2018). The parietal lobe and tool use. In G. Vallar \& H. B. Coslett (Eds.), Handbook of Clinical Neurology (3rd ed., Vol. 151, pp. 481-498). Amsterdam: Elsevier B.V. https://doi.org/10.1016/B978-0-444-63622-5.00025-5

Marcus, G. F. (2006). Cognitive architecture and descent with modification. Cognition, 101(2), 443-465. https://doi.org/10.1016/j.cognition.2006.04.009

Marcus, G. F., Rabaglia, C. D., \& Rabagliati, H. (2006). Modularity and descent-withmodification. In C. Boeckx \& K. K. Grohmann (Eds.), The Cambridge Handbook of Biolinguistics (pp. 326-340). Cambridge: Cambridge University Press. https://doi.org/10.1017/CBO9780511980435.019

Marsden, C. D., \& Obeso, J. A. (1994). The functions of the basal ganglia and the paradox of stereotactic surgery in Parkinson's disease. Brain, 117, 877-897.

Martins, M. J. D., Fischmeister, F. P. S., Gingras, B., Bianco, R., Puig-Waldmueller, E., Villringer, A., ... Beisteiner, R. (2020). Recursive music elucidates neural mechanisms supporting the generation and detection of melodic hierarchies. Brain Structure and Function, 225(7), 1997-2015. https://doi.org/10.1007/s00429-020-02105-7

Marvel, C. L., Morgan, O. P., \& Kronemer, S. I. (2019). How the motor system integrates with working memory. Neuroscience \& Biobehavioral Reviews, 102, 184-194. https://doi.org/10.1016/j.neubiorev.2019.04.017

Matchin, W. G. (2018). A neuronal retuning hypothesis of sentence-specificity in Broca's area. Psychonomic Bulletin \& Review, 25(5), 1682-1694. https://doi.org/10.3758/s13423-017$1377-6$

Matchin, W. G., \& Hickok, G. (2020). The cortical organization of syntax. Cerebral Cortex, 30(3), 1481-1498. https://doi.org/10.1093/cercor/bhz180 
Matsuzawa, T. (1991). Nesting cups and metatools in chimpanzees. Behavioral and Brain Sciences, 14, 570-571.

Miller, E. K., \& Buschman, T. J. (2008). Rules through recursion: How interactions between the frontal cortex and basal ganglia may build abstract, complex rules from concrete, simple ones. In S. A. Bunge \& J. D. Wallis (Eds.), Neuroscience of Rule-Guided Behavior (pp. 419-440). New York: Oxford University Press. https://doi.org/10.1093/acprof:oso/9780195314274.003.0022

Miller, E. K., \& Cohen, J. D. (2001). An integrative theory of prefrontal cortex function. Annual Review of Neuroscience, 24(1), 167-202. https://doi.org/10.1146/annurev.neuro.24.1.167

Miller, G. A., Galanter, E., \& Pribram, K. H. (1960). Plans and the structure of behavior. New York: Holt, Rinehart and Winston.

Oberecker, R., \& Friederici, A. D. (2006). Syntactic event-related potential components in 24month-olds' sentence comprehension. NeuroReport, 17(10), 1017-1021. https://doi.org/10.1097/01.wnr.0000223397.12694.9a

Oberecker, R., Friedrich, M., \& Friederici, A. D. (2005). Neural correlates of syntactic processing in two-year-olds. Journal of Cognitive Neuroscience, 17(10), 1667-1678. https://doi.org/10.1162/089892905774597236

Orpella, J., Mas-Herrero, E., Ripollés, P., Marco-Pallarés, J., \& de Diego-Balaguer, R. (2021). Statistical learning as reinforcement learning phenomena. BioRxiv. https://doi.org/10.1101/2021.01.28.428582

Orpella, J., Ripollés, P., Ruzzoli, M., Amengual, J. L., Callejas, A., Martinez-Alvarez, A., ... de Diego-Balaguer, R. (2020). Integrating when and what information in the left parietal lobe allows language rule generalization. PLOS Biology, 18(11), e3000895. https://doi.org/10.1371/journal.pbio.3000895 
Pallier, C., Devauchelle, A.-D., \& Dehaene, S. (2011). Cortical representation of the constituent structure of sentences. Proceedings of the National Academy of Sciences of the United States of America, 108, 2522-2527. https://doi.org/10.1073/pnas.1018711108

Papitto, G., Friederici, A. D., \& Zaccarella, E. (2020). The topographical organization of motor processing: An ALE meta-analysis on six action domains and the relevance of Broca's region. NeuroImage, 206, 116321. https://doi.org/10.1016/j.neuroimage.2019.116321

Parvizi, J. (2009). Corticocentric myopia: Old bias in new cognitive sciences. Trends in Cognitive Sciences, 13(8), 354-359. https://doi.org/10.1016/j.tics.2009.04.008

Patel, A. D. (2003). Language, music, syntax and the brain. Nature Neuroscience, 6(7), 674681. https://doi.org/10.1038/nn1082

Patel, A. D. (2008). Music, language, and the brain. Oxford: Oxford University Press.

Patel, A. D., Gibson, E., Ratner, J., Besson, M., \& Holcomb, P. J. (1998). Processing syntactic relations in language and music: An event-related potential study. Journal of Cognitive Neuroscience, 10(6), 717-733. https://doi.org/10.1162/089892998563121

Patel, A. D., Iversen, J. R., Wassenaar, M., \& Hagoort, P. (2008). Musical syntactic processing in agrammatic Broca's aphasia. Aphasiology, 22(7-8), 776-789. https://doi.org/10.1080/02687030701803804

Peretz, I., Kolinsky, R., Tramo, M., Labrecque, R., Hublet, C., Demeurisse, G., \& Belleville, S. (1994). Functional dissociations following bilateral lesions of auditory cortex. Brain, 117(6), 1283-1301. https://doi.org/10.1093/brain/117.6.1283

Poeppel, D., \& Embick, D. (2005). Defining the relation between linguistics and neuroscience. In A. Cutler (Ed.), Twenty-first century psycholinguistics: Four cornerstones (pp. 173189). Hillsdale: Erlbaum.

Price, C. J., \& Friston, K. J. (2005). Functional ontologies for cognition: The systematic definition of structure and function. Cognitive Neuropsychology, 22(3-4), 262-275. 
https://doi.org/10.1080/02643290442000095

Pulvermüller, F. (2005). Brain mechanisms linking language and action. Nature Reviews Neuroscience, 6(7), 576-582. https://doi.org/10.1038/nrn1706

Pulvermüller, F. (2010). Brain embodiment of syntax and grammar: Discrete combinatorial mechanisms spelt out in neuronal circuits. Brain and Language, 112(3), 167-179. https://doi.org/10.1016/j.bandl.2009.08.002

Pulvermüller, F. (2014). The syntax of action. Trends in Cognitive Sciences, pp. 219-220. https://doi.org/10.1016/j.tics.2014.01.001

Pulvermüller, F. (2018). Neural reuse of action perception circuits for language, concepts and communication. Progress in Neurobiology, 160, 1-44. https://doi.org/10.1016/j.pneurobio.2017.07.001

Pylkkänen, L. (2019). The neural basis of combinatory syntax and semantics. Science, 366(6461), 62-66. https://doi.org/10.1126/science.aax0050

Rauschecker, J. P., \& Scott, S. K. (2009). Maps and streams in the auditory cortex: Nonhuman primates illuminate human speech processing. Nature Neuroscience, 12(6), 718-724. https://doi.org/10.1038/nn.2331

Roy, A. C., Curie, A., Nazir, T., Paulignan, Y., des Portes, V., Fourneret, P., \& Deprez, V. (2013). Syntax at hand: Common syntactic structures for actions and language. PLoS ONE, 8(8), e72677. https://doi.org/10.1371/journal.pone.0072677

Sammler, D., Novembre, G., Koelsch, S., \& Keller, P. E. (2013). Syntax in a pianist's hand: ERP signatures of embodied syntax processing in music. Cortex, 49(5), 1325-1339. https://doi.org/10.1016/j.cortex.2012.06.007

Skeide, M. A., \& Friederici, A. D. (2016). The ontogeny of the cortical language network. Nature Reviews Neuroscience, 17(5), 323-332. https://doi.org/10.1038/nrn.2016.23

Slevc, L. R., Faroqi-Shah, Y., Saxena, S., \& Okada, B. M. (2016). Preserved processing of 
musical structure in a person with agrammatic aphasia. Neurocase, 22(6), 505-511. https://doi.org/10.1080/13554794.2016.1177090

Slevc, L. R., \& Okada, B. M. (2015). Processing structure in language and music: A case for shared reliance on cognitive control. Psychonomic Bulletin \& Review, 22(3), 637-652. https://doi.org/10.3758/s13423-014-0712-4

Slevc, L. R., Rosenberg, J. C., \& Patel, A. D. (2009). Making psycholinguistics musical: Selfpaced reading time evidence for shared processing of linguistic and musical syntax. Psychonomic Bulletin \& Review, 16(2), 374-381. https://doi.org/10.3758/16.2.374

Smalle, E. H. M., Daikoku, T., Szmalec, A., Duyck, W., \& Möttönen, R. (2022). Unlocking adults' implicit statistical learning by cognitive depletion. Proceedings of the National Academy of Sciences, 119(2), 1-9. https://doi.org/10.1073/pnas.2026011119

Steinbeis, N., \& Koelsch, S. (2008). Shared neural resources between music and language indicate semantic processing of musical tension-resolution patterns. Cerebral Cortex, 18(5), 1169-1178. https://doi.org/10.1093/cercor/bhm149

Stocco, A. (2019). Commentary on Neuroemergentism: A framework for studying cognition and the brain. The neurocomputations of neuroemergentism: Long-term memory + reinforcement learning = language $?$ Journal of Neurolinguistics, 49, 248-251. https://doi.org/10.1016/j.jneuroling.2018.04.008

Summerfield, C., Luyckx, F., \& Sheahan, H. (2020). Structure learning and the posterior $\begin{array}{llllll}\text { parietal } & \text { cortex. } & \text { Progress in Neurobiology, } & 184,\end{array}$ https://doi.org/10.1016/j.pneurobio.2019.101717

Thibault, S., Py, R., Gervasi, A. M., Salemme, R., Koun, E., Lövden, M., ... Brozzoli, C. (2021). Tool use and language share syntactic processes and neural patterns in the basal ganglia. Science, 374(6569). https://doi.org/10.1126/science.abe0874

Tuckute, G., Paunov, A., Kean, H., Small, H., Mineroff, Z., Blank, I., \& Fedorenko, E. (2022). 
Frontal language areas do not emerge in the absence of temporal language areas: A case study of an individual born without a left temporal lobe. Neuropsychologia, 169, 108184. https://doi.org/10.1016/j.neuropsychologia.2022.108184

Uddén, J., \& Bahlmann, J. (2012). A rostro-caudal gradient of structured sequence processing in the left inferior frontal gyrus. Philosophical Transactions of the Royal Society of London. Series B, Biological Sciences, 367(1598), 2023-2032. https://doi.org/10.1098/rstb.2012.0009

Ullman, M. T. (2006). Is Broca's area part of a basal ganglia thalamocortical circuit? Cortex, 42(4), 480-485. https://doi.org/10.1016/S0010-9452(08)70382-4

Ullman, M. T., Earle, F. S., Walenski, M., \& Janacsek, K. (2020). The neurocognition of developmental disorders of language. Annual Review of Psychology, 71(1), 389-417. https://doi.org/10.1146/annurev-psych-122216-011555

Zaccarella, E., Papitto, G., \& Friederici, A. D. (2021). Language and action in Broca's area: Computational differentiation and cortical segregation. Brain and Cognition, 147, 105651. https://doi.org/10.1016/j.bandc.2020.105651 